\title{
Group physiotherapy compared to individual physiotherapy to treat urinary incontinence in aging women: study protocol for a randomized controlled trial
}

\author{
Chantale Dumoulin ${ }^{1 *}$, Mélanie Morin ${ }^{2}$, Marie-Hélène Mayrand ${ }^{3}$, Michel Tousignant $^{4}$ and Michal Abrahamowicz ${ }^{5}$
}

\begin{abstract}
Background: Urinary incontinence $(\mathrm{UI})$, one of the most prevalent health concerns confronting women aged over 60 years, affects up to $55 \%$ of older community-dwelling women-20-25\% with severe symptoms. Clinical practice guidelines recommend individualized pelvic floor muscle training (PFMT) as a first-line treatment for stress or mixed $\mathrm{UI}$ in women, although lack of human and financial resources limits delivery of this first-line treatment. Preliminary data suggest that group-based treatments may provide the answer. To date, no adequately powered trials have evaluated the effectiveness or cost-effectiveness of group compared to individual PFMT for UI in older women. Given demographic projections, high prevalence of UI in older women, costly barriers, and group PFMT promising results, there is a clear need to rigorously compare the short- and long-term effectiveness and cost-effectiveness of group vs individual PFMT.
\end{abstract}

Methods/Design: The study is designed as a non-inferiority randomized controlled trial, conducted in two facilities (Montreal and Sherbrooke) in the Canadian province of Quebec. Participants include 364 ambulatory, communitydwelling women, aged 60 years and older, with stress or mixed UI. Randomly assigned participants will follow a 12-week PFMT, either in one-on-one sessions or as part of a group, under the supervision of a physiotherapist. Blinded assessments at baseline, immediately post intervention, and at one year will include the seven-day bladder diary, the 24-h pad test, symptoms and quality of life questionnaires, adherence and self-efficacy questionnaire, pelvic floor muscle function, and cost assessments. Primary analysis will test our main hypothesis that group-based treatment is not inferior to individualized treatment with respect to the primary outcome: relative (\%) reduction in the number of leakages.

Discussion: Should this study find that a group-based approach is not less effective than individual PFMT, and more cost-effective, this trial will impact positively continence-care accessibility and warrant a change in clinical practice.

Trial registration: ClinicalTrials.gov, NCT02039830. Registered on 12 December 2013; Study protocol version 2; 21 November 2013.

Keywords: Urinary incontinence, Pelvic floor muscle training, Elderly women

\footnotetext{
* Correspondence: chantal.dumoulin@umontreal.ca

${ }^{1}$ School of Rehabilitation, Faculty of Medicine, Université de Montréal,

Research Centre of the Institut Universitaire de Gériatrie de Montréal, 4565

Queen Mary M-5816, Montreal, QC H3W 1W5, Canada

Full list of author information is available at the end of the article
} 


\section{Background}

Urinary incontinence (UI), one of the most prevalent health concerns confronting women aged 60 years and over, affects up to $55 \%$ of older community-dwelling women [1]-20-25\% having severe symptoms (> $10 \mathrm{UI}$ episodes/week) [1]. Recognized as a serious medical condition, UI is also a social issue, one that engenders shame and negative self-perception leading to reduced social interaction and physical activity [2-4]. It is associated with poor self-rated health, impaired emotional and psychological wellbeing, and impaired sexual relationships $[3,5,6]$. It doubles women's risk of being admitted to a nursing home, independently of age or the presence of any other co-morbid conditions [7].

Clinical practice guidelines recommend individualized pelvic floor muscle training (PFMT) as a first-line treatment for stress or mixed UI in women (Level A evidence [8-12]); however, inadequate financial and human resources prevent delivery of individualized PFMT in many countries [13-15].

Evidence from two recent randomized controlled trials (RCTs) suggests that group PFMT is effective for treating stress and mixed UI in older women, resulting in high continence rates post-intervention compared to no treatment or bladder training $[16,17]$. A group-delivery approach offers an effective way to overcome financial and human resource barriers; moreover, it has been shown to increase participant's motivation, adherence, and UI self-management capabilities [18-22]. In recent years, the search for less costly forms of rehabilitative treatments has taken on a greater impetus and been subject to intense debate $[8,23,24]$. It is now recognized that for some pathological conditions, group rehabilitation approaches offer a viable solution, one that would permit better allocation of available economic resources-material and human [23]. Additionally, group intervention is already acknowledged, within the field of health promotion, as a powerful tool for promoting behavior modification: group sessions provide greater motivation (by reducing an individual's sense of isolation) and a forum for providing information (particularly for those too timid to ask questions) and foster peer support and discussions: e.g. Weight Watchers [25]. Tackling UI through group sessions could also prove to be an effective means of educating and encouraging active selfmanagement; therefore, it could have an impact in the longer term. [26, 27] To date, no studies have compared with adequate power the long-term effectiveness or costeffectiveness of group PFMT to individualized PFMT for the treatment of UI in older women [18].

Given group PFMT's promising results, there is a clear need to compare the short- and long-term effectiveness and cost-effectiveness of group vs individual PFMT, especially considering demographic projections, the high prevalence of UI in older women and the costly barriers to individualized PFMT. If we demonstrated that groupbased treatment is not meaningfully less effective than individualized one-on-one treatment, and more costeffective, group-based PFM training would be warranted as a first-line UI treatment.

\section{Rationale for a non-inferiority trial}

A non-inferiority design was chosen because: (1) individual PFMT is the standard of care; (2) recent literature and our preliminary data on aging women suggest that group-based PFMT may be effective immediately post treatment and in the short term (less than six months); (3) there is potential for long-term benefits from group-based interventions resulting from increased peer-support and mutual self-help leading to increased compliance with treatment; (4) the anticipated lower cost; and (5) the potential to improve accessibility to care through a group approach (overcoming lack of human and financial resources). Should this study find that a group-based PFM training approach is not meaningfully less effective than individual PFM training, it would warrant a change in clinical practice.

\section{Objectives}

The overall objective of the GROUP (Group Rehabilitation Or IndividUal Physiotherapy for Urinary Incontinence in Aging Women) trial is to determine if group-based PFMT for women aged 60 years and older with stress or mixed UI is not meaningfully less effective, sustainable, and affordable than the currently recommended individualized (one-on-one) PFMT.

The specific objectives are to compare the effectiveness of group-based PFMT vs individualized PFMT on:

1. the $\%$ reduction in the number of UI episodes, as measured by the seven-day diary [28], immediately post intervention, and at one year post randomization (primary outcome);

2. lower urinary track symptoms, level of distress, and quality-of-life impact, immediately post intervention and at one year post randomization. These secondary outcomes will be measured by, respectively, the sevenday bladder diary (number of urinary incontinence, number of micturition [28], the 24-h pad test (quantity of urine loss) [29], five modules of the International Consultation on Incontinence Questionnaire (ICIQ): the ICIQ-Urinary Incontinence short form (ICIQ-UI short form) [30], the ICIQNocturia (ICIQ-N) [31], the ICIQ-Lower Urinary Tract Symptoms quality of life (ICIQ-LUTSquol) [32], the ICIQ-Vaginal symptoms (ICIQ-VS) [33], the ICIQ-Female Lower Urinary Tract Symptoms sex (ICIQ-FLUTSex) [34]; 
3. self-efficacy, immediately post intervention and at one year post randomization, as measured by the Geriatric Self Efficacy scale [35] and the Broom Self Efficacy questionnaire Part A [36];

4. impression of improvement and benefits, immediately post intervention and at one year post randomization as measured by the Patient Global Impression of Improvement (PGI-I) [37] and the Satisfaction and willingness to have another treatment (B\&W) [17];

5. PFM strength, function, and morphometry as measured by the digital Oxford scale [38], pelvic floor dynamometry [39], transperineal US [40] immediately post intervention and at one year post randomization.

We will also compare the direct costs of group-based PFMT and individualized PFMT using the adapted Dowell-Bryant Incontinence Cost Index (DBICI) [41] immediately post intervention and at one year post randomization.

\section{Methods}

Trial design

The present study is a non-inferiority RCT involving two metropolitan areas in the province of Quebec, Canada: Montreal and Sherbrooke. This trial design is based on recommendations outlined in the "Research Methodology" chapter of the International Consultation on Incontinence book [42]. It comprises three parallel evaluations at, respectively, pre-intervention, post-intervention (1-2 weeks after treatment will have been completed), and one year post randomization, each involving structured interviews, questionnaires, bladder diary, pad tests, as well as physical and gynecological exams (Fig. 1).

\section{Research sites}

The study is conducted at the research center of the Institut Universitaire de Gériatrie de Montréal and at the research center of the Centre Hospitalier de l'université de Sherbrooke.

\section{Participants}

The target population is older women with stress UI and mixed UI. "Older" is defined as aged 60 years and over; as a cut-off age, this population of post-menopausal women have a UI profile distinctive from that of pre-menopausal or post-partum women [43]. Other RCTs on UI in older women also employ this cut-off age $[17,44]$. There is no upper age limit as women as old as 98 years have been cured of UI through PFMT [45]. Participants are classified as incontinent if they report a weekly average of three or more episodes of involuntary urine loss during the preceding three months. This is a validated indicator of UI that has been used in cohort studies and RCTs on UI $[16,44]$.
Finally, type of UI is confirmed as a pattern of stress/ mixed UI on the validated Questionnaire for Incontinence Diagnosis (QUID) [46].

\section{Recruitment}

Women are being recruited from community advertisements, newspaper ads, the Research Center of the Institut Universitaire de Gériatrie de Montréal's bank of participants, gynecology and urology clinics within the metropolis of the two study centers. In Montreal, these include the gynecology clinic at the Maisonneuve-Rosemont Hospital, and the CHUM gynecology clinics, the CLSC Lucille Teasdale, the CSSS Jeanne Mance. In Sherbrooke, this includes the urology and gynecology clinics at the Centre Hospitalier Universitaire de Sherbrooke. A poster explaining the research project is being displayed at each clinic. Interested candidates are invited to call the research coordinator.

Determination of eligibility is achieved in two steps: (1) telephone eligibility evaluation with a research assistant; and (2) on-site eligibility evaluation with the evaluator. Participant eligibility criteria are described in Table 1. These criteria ensure recruitment of a homogeneous sample of community-based older women with stress/mixed urinary incontinence signs and symptoms. Women excluded from the study are referred back to their healthcare provider to receive standard incontinence care.

\section{Telephone eligibility evaluation (20 min)}

Initial contact is over the phone. A research assistant briefly explains the project. Potential candidates are informed of the study's objectives and procedures. If interested, they are screened for eligibility using a standardized telephone questionnaire developed and used in our previous studies. The UI type is determined by the QUID over the telephone; it is a six-item self-administered questionnaire developed to identify and differentiate UI types in women [46]. This instrument has shown good internal consistency and test-retest reliability, good content and criterion validity, and good specificity/sensitivity in women with UI [47]. Further, QUID is highly recommended for UI type classification by the International Consultation on Incontinence (Grade A) [47]. When women present with stress or mixed UI, the consent form (Additional file 1) is mailed out to the participant with the seven-day bladder diary $[28,47]$; the diary is completed and brought to the evaluator at the onsite eligibility evaluation.

\section{Onsite eligibility evaluation ( $1 \mathrm{~h}$ )}

Women are scheduled for onsite evaluations with a physiotherapist if they report at least three UI episodes on the seven-day bladder diary. After signing the consent form, the Mini-Mental State Examination [48] questionnaire is completed (excludes women with cognitive 


\begin{tabular}{|c|c|c|c|c|c|c|c|}
\hline \multirow[b]{2}{*}{ TIMEPOINT } & \multirow{2}{*}{$\begin{array}{c}\text { Enrolment } \\
\text { September } \\
2012 \text { to } \\
\text { March } \\
2017 \\
\end{array}$} & \multirow{2}{*}{$\begin{array}{c}\text { Allocation } \\
0 \mathrm{~m}\end{array}$} & \multicolumn{5}{|c|}{$\begin{array}{l}\text { Post-allocation- Intervention delivery and } \\
\text { research follow up }\end{array}$} \\
\hline & & & $1 m$ & $3 m$ & $6 m$ & $9 m$ & $12 m$ \\
\hline \multicolumn{8}{|l|}{ ENROLMENT: } \\
\hline \multirow{4}{*}{$\begin{array}{r}\text { Telephone eligibility screen } \\
\text { On site eligibility screen } \\
\text { Informed consent } \\
\text { Allocation }\end{array}$} & $x$ & & & & & & \\
\hline & & $x$ & & & & & \\
\hline & & $x$ & & & & & \\
\hline & & $x$ & & & & & \\
\hline \multicolumn{8}{|l|}{ INTERVENTIONS: } \\
\hline \multicolumn{8}{|l|}{ Individual PFMT } \\
\hline \multicolumn{8}{|l|}{ Group PFMT } \\
\hline \multicolumn{8}{|l|}{ ASSESSMENTS: } \\
\hline \multicolumn{8}{|l|}{$\begin{array}{r}\text { Basic socio-demographic } \\
\text { data }\end{array}$} \\
\hline BMI & & $x$ & & & & & \\
\hline \multirow{3}{*}{ 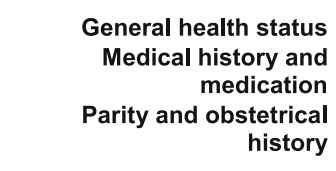 } & & $x$ & & & & & \\
\hline & & $x$ & & & & & \\
\hline & & $x$ & & & & & \\
\hline \multirow{2}{*}{$\begin{array}{l}\text { Type and duration of UI } \\
\text { 7-day bladder diary }\end{array}$} & & $x$ & & & & & \\
\hline & & $x$ & & $x$ & & & $x$ \\
\hline $24 h$ pad test & & $x$ & & $x$ & & & $x$ \\
\hline \multirow{3}{*}{$\begin{array}{r}\text { ICIQ modules } \\
\text { Geriatric self-efficacy index } \\
\text { Broom self-efficacy index }\end{array}$} & & $x$ & & $x$ & & & $x$ \\
\hline & & $x$ & & $x$ & & & $x$ \\
\hline & & & & $x$ & & & $x$ \\
\hline \multirow{3}{*}{$\begin{array}{r}\text { PGI-I } \\
\text { PFM strength, } \\
\text { morphometry and function } \\
\text { Costs related to } \\
\text { interventions }\end{array}$} & & & & $x$ & & & $x$ \\
\hline & & $x$ & & $x$ & & & $x$ \\
\hline & & $x$ & & $x$ & & & $x$ \\
\hline \multirow{2}{*}{$\begin{array}{r}\text { Treatment satisfaction } \\
\text { Exercise maintenance } \\
\text { questionnaire }\end{array}$} & & & & $x$ & & & $x$ \\
\hline & & & & & $x$ & $\mathrm{x}$ & $x$ \\
\hline Adverse events & & & $x$ & $x$ & $x$ & $x$ & $x$ \\
\hline
\end{tabular}

Fig. 1 Schedule of enrolment, interventions, and assessments

impairment) and a vaginal exam is conducted to identify any perineal pain or prolapse likely to interfere with either the evaluation or the intervention. Those not eligible for the present study are referred back to their healthcare provider. Eligible participants complete the evaluation and are randomized to either individualized or group PFMT.

\section{Interventions}

The women in both groups receive the same 12-week PFMT, either in one-on-one sessions or as part of a group, under the direction of an experienced physiotherapist trained in pelvic floor rehabilitation. The choice of a 12week PFMT approach is based on muscle physiology theory: strength-training programs show positive effect after 8-12 weeks [49]. In post-menopausal women, Gunnarsson et al. reported a significant improvement in strength and reduction in UI episodes after 12 weeks of training [50]. Both the one-on-one and group-based PFMT sessions are conducted once a week; participants are deemed to have successfully completed the program if they attended ten or more of the 12 sessions. Although there is no known side effect/complication related to PFMT other than possible discomfort following the intervention, any adverse event is monitored. All participants are instructed to contact research personnel should they experience any adverse event at any point during the study. 
Table 1 Inclusion and exclusion criteria

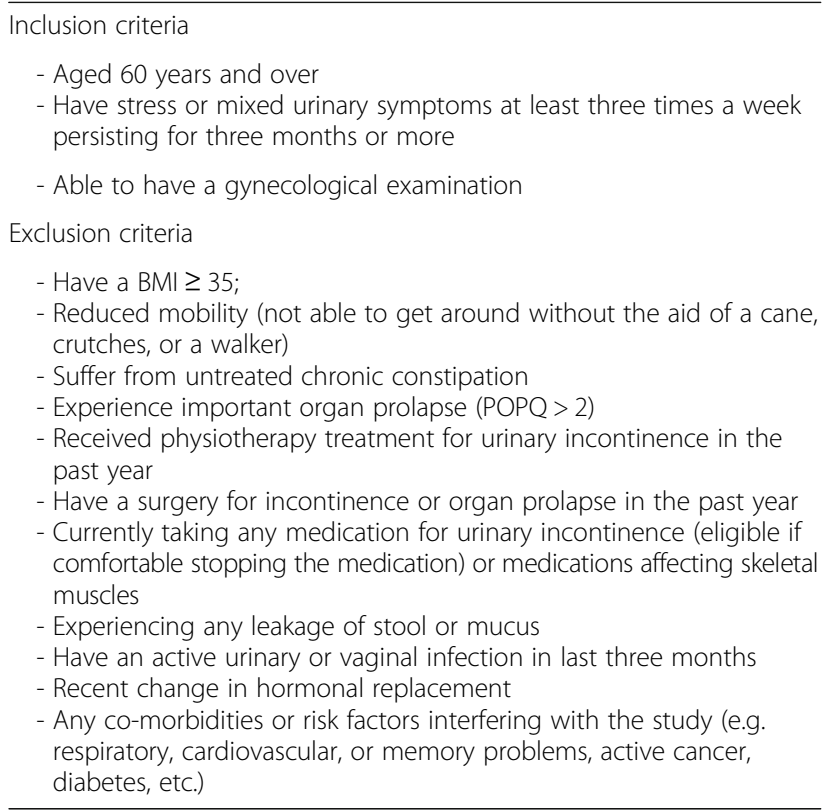

\section{PFMT sessions}

For both groups, the weekly sessions last $1 \mathrm{~h}$ and include a 15 -min educational period and a 45 -min exercise component. The educational period covers the various functions of the PFMs, including pre-contraction and normal bladder control, and voiding parameters: fluids and fluid intake; toilet positions; and voiding dynamics. The exercise component includes PFM strength, endurance, and coordination exercises. Between PFM exercises, lower extremity strength, balance and functional exercises (dance) are performed. This approach is in line with our previous research findings, which indicate that mixed and stress UI older women have reduced PFM and lowerextremity strength and balance as compared to continent women $[18,51,52]$. It is also a component of PFMT programs designed for the elderly $[16,17]$. The treatment protocol is divided into three phases allowing for gradual progression in treatment; that is, the gradual addition of increasingly difficult exercises in terms of exercise duration, repetition, and position. Each phase lasts four weeks. See Table 2 for monthly treatment's exercise details.

\section{Individualized PFMT}

The only specificity of this treatment group is that women participate in one-on-one, 1 -h sessions with intra-vaginal electomyography (EMG) biofeedback under the supervision of an experienced physiotherapist. This treatment approach is in line with clinical practice in Canada [53].

\section{Group-based PFMT}

The only specificity for this treatment group is that women participate in groups of eight in a weekly 1-h session, under the supervision of an experienced physiotherapist. They are also offered up to three optional private $(20 \mathrm{~min})$ sessions with the physiotherapist leading their group (either before or after a group session) to ensure understanding and correct performance of a PFM contraction - as confirmed by vaginal digital palpation [17]. This treatment approach is consistent with Bo's PFM exercise class [54] and our more recent pilot cohort studies indicating it is both feasible and effective intervention for UI in aging women $[18,55]$. In case of slow recruitment, we have established a minimum group size of six participants in order to avoid delays for those assigned to group physiotherapy.

\section{Home PFMT program}

Women in both groups are expected to perform PFM strengthening, endurance, and coordination exercises at home, five days a week, for the duration of the treatment $[18,22,51,55]$. To support progression in the treatment, the home exercise program parallels the three phases in the treatment protocol with the gradual addition of increasingly difficult exercises every four weeks. To standardize home PFM exercises, each participant is given a PFM exercise diary describing the home PFMT exercises, in which they can record their adherence to the home program. Finally, all participants are asked to refrain from seeking other forms of treatment (such as medication or surgery) during the study.

\section{Standardization of treatment}

Physiotherapists who deliver the interventions are extensively trained in standardized treatment protocols and rigorous procedures to conduct both individualized and group-based PFMT. Each physiotherapist participates in a 4-h training workshop, is given a written a treatment protocol with checklist, and is individually supervised for three to six treatments. Routinely, during the course of the study, all physiotherapists come together with the study team to ensure that consistency in the protocol is being maintained and to discuss concerns that may arise. For physiotherapist effect not to confound results, the same physiotherapist is conducting both individualized and group-based PFM training in a single location.

\section{Randomization/blinding}

\section{Sequence generation and allocation concealment}

To prevent imbalance on important patient characteristics while ensuring equal sizes of the two trial arms, we used stratified randomization with random blocks within each stratum. Specifically, participant allocation is stratified by (1) center (Montreal and Sherbrooke); and then (2) within each center, by UI type (MUI and SUI). Within each of the four (center-by-UI type) resulting strata, the randomization sequence was generated 


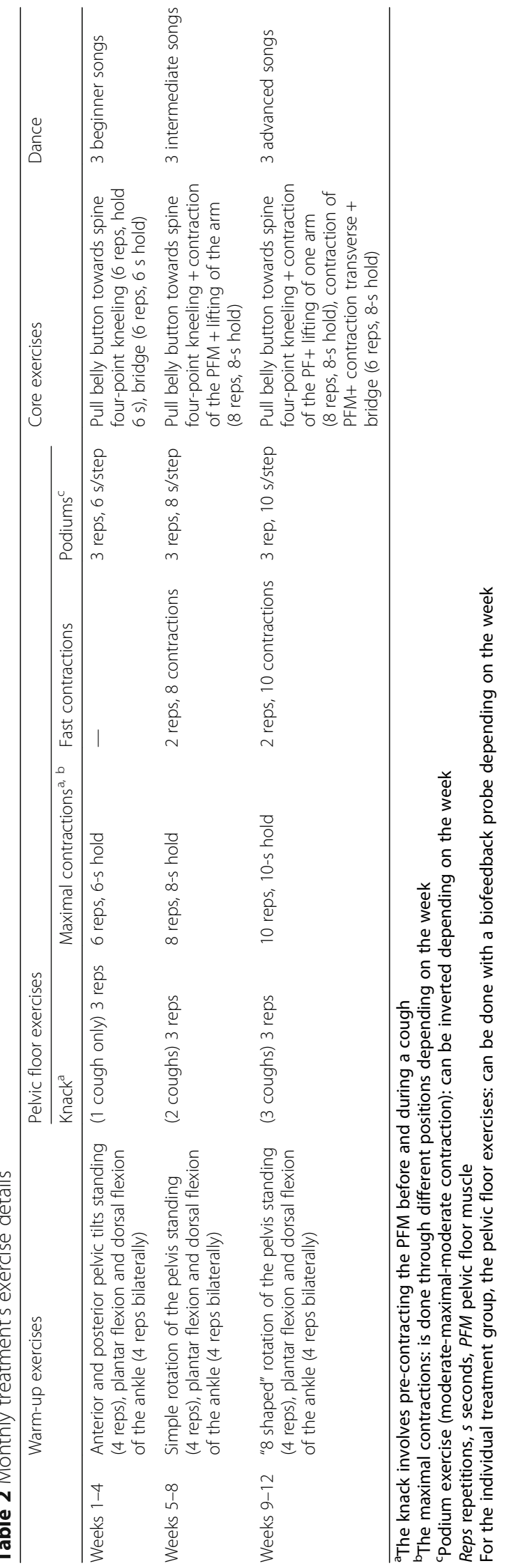


through a computerized system, by a CRIUGM statistician (FG), before the trial to create random permutated blocks of varying sizes (4-6), making the particular sequences difficult to predict. Randomization process takes place after a participant's initial evaluation and written consent. Randomization lists are then used, by an independent individual (CRIUGM IT service analyst), to assign eligible participants to one of two trial arms.

\section{Intervention allocation}

A research assistant (one in each center) communicates with the CRIUGM IT service analyst to get the next sequential allocation and inform the participants as to which treatment they have been allocated and, in addition, organize the logistics of the PFMT intervention.

\section{Blinding}

Investigators, data analysts, and physiotherapists in charge of outcome evaluations remain blinded to the individual participants trial group allocation. Although participants cannot be blinded to their own group allocation, they are blinded to the study's hypothesis and to the treatment offered in the alternate group. Despite their different formats, both groups have parallel weekly sessions in terms of content and time, but at different locations and/or on different days. To minimize the risk of assessor's unblinding, participants are asked (both in the consent form and at the time of each assessment) not to discuss their treatment with the independent assessor. In addition, in each center, the assessment is separated from the intervention session, by both time and location.

\section{Outcome measures and moderators/confounders Primary outcome measure}

The primary outcome measure is the mean percent reduction in the total number of UI episodes one year post randomization, as measured by a seven-day bladder diary [28]. For example, a participant experiencing four leaks per week pre intervention and two leaks per week at the one-year follow-up would be considered to have had a $50 \%$ reduction in leakage episodes. The number of leaks (as measured by the seven-day diary) is considered one of the most reliable measures of success for incontinence treatment and has been widely used in this type of research [16, 28, 44, 47, 51]. Moreover, the reduction in the number of daily leaks, expressed as a percentage, is information that can be easily understood by participants in pre-treatment counseling. As a measurement tool, the seven-day diary has a high compliance rate and good reproducibility $[28,56]$.

\section{Secondary outcome measures}

Several secondary outcomes are assessed in this study, in line with the recommendations of the 4th International
Consultation on Incontinence [57] and the International Continence Society [58]. For each outcome, we have selected measurement instruments that have highest psychometric properties (validity, reliability, and response to change).

1. The seven-day bladder diary: The number of micturition per day/night is monitored to document lower urinary frequency during the day and nocturia [28].

2. The 24-h pad test: This is a validated measure of quantity of urine leakage in $24 \mathrm{~h}$, as measured by the weight of the pads used during a 24-h period minus the weight of the pads before the test. It is identified as a realistic appraisal of the typical urine loss during ordinary daily activity [29]. The upper limit of "normal" for the 24-h pad test has been defined for continent women as being $1.3 \mathrm{~g}$ [47].

3. The International Consultation on Incontinence Questionnaire (ICIQ) modules: Five ICIQ modules, known to provide brief and robust measures of UI symptoms, quality of life, and outcome of treatment are used [32].

i. The ICIQ-Urinary Incontinence short form: a four-item questionnaire which evaluates the impact of symptoms of incontinence on quality of life and outcome of treatment (0-21 overall score, with greater values indicating increased severity) [30].

ii. The ICIQ-Nocturia: a two-item questionnaire which evaluates the impact of symptoms of nocturia on quality of life and outcome of treatment ( $0-8$ overall score, with greater values indicating increased symptom severity) [31].

iii. The ICIQ-Vaginal Symptoms: a 14-item questionnaire which evaluates the impact of vaginal symptoms and associated sexual matters on quality of life and outcome of treatment (0-53 vaginal symptoms subscale, $0-58$ sexual matters subscale, $0-10$ overall impact on quality of life subscale; with greater values indicating increasing problems) [33].

iv. The ICIQ-Female Lower Urinary Tract Symptoms sex: a four-item questionnaire for evaluating sexual matters associated with female lower urinary tract symptoms $(0-14$ overall score, with greater values indicating increasing problems with sexual matters) [34].

v. The ICIQ-Lower Urinary Tract Symptoms quality of life: a 20-item questionnaire which evaluates quality of life in urinary incontinent patients $(0-14$ overall score with greater values indicating increasing problems). The ICIQLUTSqol provides a detailed and robust 
measure to assess the impact of urinary incontinence on quality of life with particular reference to social effects [32].

4. The geriatric self-efficacy index: A 20-item instrument that enables measurement of whether a person is confident in their ability to prevent urine loss. It has been shown to be a reliable and valid instrument in the aging female population with UI [35].

5. The Broom self-efficacy index (Part A): A 14-item questionnaire to evaluate women's confidence in performing pelvic floor muscle exercise [36]. The Broom self-efficacy index has solid psychometric properties and is a useful tool to measure selfefficacy in doing PFM exercises [59].

6. Patient global impression of improvement PGI-I: a single-item global index used to measure improvement in urinary continence following PFMT on a 7-point scale that ranges from "very much better" to "very much worse." The PGI-I has shown acceptable convergent and discriminant validity for measuring outcomes in studies of behavioral treatment for UI [37, 60].

7. Satisfaction with treatment: A single-item tool was used to document and capture perceived satisfaction with treatment: "satisfied" (does not need other treatments); "unsatisfied" (would like another treatment for UI) [17].

8. PFM strength, morphometry and function:

i. PFM strength on Oxford scale (digital palpation):

The Oxford scale is a 5-point scale used for

PFM strength assessment amongst

physiotherapists. Laycock and Jerwood have established intra-therapist reliability of this scale, tested in bent-knee lying $[38,61]$.

ii. PFM function: An intravaginal dynamometric speculum, designed by members of our research team is used to measure passive (tone) and active forces (strength), speed of contraction, coordination with cough and endurance [39]. This instrument has been widely assessed for its psychometric properties including its reliability, validity, and responsiveness [39, 62].

iii. PFM Morphometry: A Siemens Acuson Antares system with a 3-5-MHz curvilinear 3D/4D probe (in Montreal) and a GE Voluson Expert system with a $2-6-\mathrm{MHz}$ curvilinear 3D/4D probe (in Sherbrooke) is used to evaluate several morphometric parameters at rest, during PFM contraction and on effort (cough and Valsalva): levator hiatus area and diameter, bladder neck position and displacement as well as levator plate height, using a validated and reliable methodology $[40,63,64]$.

\section{The modified Dowel-Bryant Incontinence Cost index}

Costs related to interventions The DBICI, a universally applicable questionnaire, has been adapted to measure the intervention costs for the two treatment groups $[41,65]$. The validated DBICI has been used in RCTs with community-dwelling populations of women aged 40 years and over for non-surgical UI interventions [66] and is recommended by the International Consultation on Incontinence Research Guidelines [10, 42]. Section 1 of the DBICI documents, monthly self-reported personal incontinence expenditures (disposable and reusable incontinence products), is used integrally. Section 2 deals with treatment expenditures and has been adapted as follows: (1) Treatment costs: rather than self-reported, treatment duration (in h) for each participant is based on statistics maintained by the research coordinators using a standardized form developed for a previous cost-effectiveness study [67]. The number of hours is multiplied by the mean hourly salary of the physiotherapists participating in the study. For the groupbased PFMT, the total number of hours will be divided by the number $(\mathrm{n}=8)$ of intended class participants (i.e. not actual attendance); (2) Other consultations: the frequency of visits to incontinence-related medical professionals is documented by category (general practitioners and specialists). A research assistant will phone participants at three months and six months post intervention in order to collect information. Estimated costs are based on the frequency and a mean cost per visit based on the public health system regulations for each category (Régie de l'assurance maladie du Québec). UI medication costs is excluded from the trial as those with UI medication are excluded at study entrance.

Pre-treatment covariates In order to be able to adjust for potential imbalances, across the two randomization groups, in the distributions of covariates, that may be potentially associated with the outcomes, baseline measurement of the following variables will be carried out: basic socio-demographic data including age, BMI, general health status, medical history and medications, parity and obstetrical history as well as type and duration of UI. In secondary analyses, we will then use multivariable regression models to adjust the between-groups differences in the outcomes for these covariates, in addition to the pretreatment values of the respective outcome variable.

Sample size calculation Sample size was calculated so as to ensure the adequate power and type I error rate for testing the primary hypothesis of non-inferiority of the group-based intervention relative to the individualized intervention, in achieving the relative reduction (in $\%$ ) in the number of UI episodes at one year. Sample size 
calculations followed CONSORT Guidelines for nonInferiority trials [68]. First, based on clinical relevance [69] (minimum clinically relevant difference $=10 \%$ ) and our pilot data [18-22], we set the "margin of equivalence" (i.e. the upper limit of the non-inferiority interval) as corresponding to a $10 \%$ difference between mean \% reduction in the number of UI episodes in the "standard treatment" of the individualized intervention minus the group-based intervention arm. This implies that we test the null hypothesis $\mathrm{H0}: \mathrm{d} \leq 10 \%$, where $\mathrm{d}$ denotes the true difference between the mean relative reductions in the number of UI episode in the two arms (individualized minus group-based) against the alternative hypothesis $\mathrm{H} 1: \mathrm{d}>10 \%$. According to the CONSORT guidelines, this choice of the "margin of equivalence" implies that the non-inferiority hypothesis should be rejected whenever the upper bound of the two-tailed $(1-2 \alpha) \%$ confidence interval (CI) for the difference between the two mean \% reductions exceeds $10 \%$. In the specific context of a noninferiority trial, $\alpha$ is the selected risk of a false acceptance of the non-inferiority hypothesis (based on a one-tailed CI-based test, equivalent to an independent-group t-test); i.e. of a false conclusion that a truly inferior intervention is equally efficacious as the "standard treatment" [68].

To err on the conservative side, we relied on the "standard" 95\% CI, which corresponds to a stringent one-tailed type I error rate of $\alpha=0.025(2 \alpha=1-0.95=$ $0.05)$. Similar to other recent non-Inferiority trials (e.g. $[70,71]$ ) and CONSORT guidelines [68], for the purpose of sample size calculation, we assume that the true difference between the mean reductions (\%) achieved by the two interventions will be zero (i.e. that they are equally effective). Note that this assumption of equivalence of the two interventions being compared in our trial is consistent with both (a) clinical expectations and (b) (limited) published evidence concerning young and middle-aged UI women [72-74]. Finally, both published trials, which evaluated similar interventions in older women, reported within-group standard deviations (SD) of the individual \% reduction scores of about $27 \%[44,75]$. Accordingly, we assumed SD $=27 \%$ in our calculations.

Under the above assumptions, we estimated the sample size needed to ensure high (90\%) power to demonstrate the non-inferiority of the group-based intervention (assuming, as mentioned above, that the true difference is $0 \%$ ). Thus, we calculated $\mathrm{N}$, for which the probability that the upper boundary of the two-tailed $95 \%$ CI for the difference in the mean relative reduction (Individual Group) excludes the "upper threshold of non-inferiority" (10\% difference), will reach at least $90 \%$. The sample size calculations were performed using the program in the PASS software package, designed specifically for power/ sample size estimation for non-inferiority trials [76]. Under the assumptions outlined above, we will need 155 participants per group, for a total of 310 individuals. We also need to account for possible losses to follow-up, as we expect a $15 \%$ attrition rate by the end of the one year, based on both our pilot data and similar published trials $[16,17,22,51]$. Thus, we will recruit an additional 54 participants per group, increasing the total number of participants to $364\left(364^{*}(1-0.15)=310\right)$.

\section{Trial management}

The PI and a research coordinator regularly contact (through emails, telephone, or in person) each participating urology and gynecology clinic in order to promote and monitor progress in recruitment. The PI, the evaluators, physiotherapists, research assistant, and the Sherbrooke and Montreal coordinators conduct conference calls or face-to-face meetings in order to monitor the study's progress. All members of the research team are kept informed of progress through a newsletter, every six months.

All collected data are anonymized and kept under lock and key at Dr. Dumoulin and Morin's laboratories at the research center of the Institut Universitaire de Gériatrie de Montréal and research center of the Cewntre Hospitalier de l'Université de Sherbrooke. After each assessment and on the same day, files are reviewed by the research assistant in order to identify missing data. Any missing information is retrieved immediately by research assistants directly from the study participants. Data are entered weekly - trimestrally (depending on recruitment rate) into a computerized database system SPSS data Entry 4.0 both in Sherbrooke and in Montreal centers. The database is backed up on a weekly basis. A PIN number known only to the research group is required to access the data entry computer. A final quality-control step will be taken at the time of the data analysis by the trial statistician. Frequency distributions and ranges will be analyzed to detect outliers that could signal potential errors. The data will be analyzed without any nominative identifiers.

\section{Adherence}

Based on the results of our previous feasibility study and post-study focus group, no significant problems are expected with treatment adherence in either group $[22,26]$. Indeed, participants $(n=27)$ in the feasibility study complied well with study demands in terms of attendance at the treatment sessions (90\%), completion of the daily exercise program $(78 \%)$, and data collection (95\%) [26]. Post-study focus groups identified "close supervision by the physiotherapist" and a "short daily PFM exercise program" as key facilitators in the participant's completion of a 12-week group-based PFMT program and their adherence to the daily home PFM exercise program [22]. Adherence will be 
measured as follows: the women will be provided with diaries to record home exercise adherence for the 12-week treatment session. In addition, exercise maintenance will be assessed with a standardized questionnaire, at three and six months post intervention as well as at the 12month follow-up evaluation. Additionally, adherence to each supervised weekly treatment sessions will be recorded by the physiotherapists.

To ensure evaluation and treatment adherence, we used the following strategies: (1) individual consultations using vaginal digital palpation ensure that participants can perform a PFM contraction correctly (the physiotherapist is available before and after individual and group treatment sessions to provide tips and answer questions regarding the weekly treatment and home exercise program); (2) an exercise diary is given to each participant to bring home and is verified by the physiotherapist each week throughout the treatment; (3) the home exercise program comprises a short and simple daily PFM exercise program to facilitate exercise practice; (4) women in both groups are reminded of their appointments (evaluation and treatments) by a research assistant. They are scheduled at participants' convenience; (5) follow-up contact calls are made at three and six months post intervention to maintain contact. Evaluators solicit information from participants on their intention to change address or telephone number and on the frequency of visits to incontinence-related medical professionals. They also verify if medications have been prescribed, surgery conducted, and if there are any other health problems likely to influence UI. Finally, they monitor their continued adherence to PFMT; and (6) participants are reimbursed for their travel and parking expenses related to participation in the study.

\section{Data analysis}

General analytical strategy: as opposed to conventional superiority trials, in non-inferiority trials per-protocol analysis is generally preferable to intention-to-treat (ITT) analysis, because the postulated hypothesis being tested assumes no difference between the two interventions [68]. Indeed, in non-inferiority trials, a "conservative" approach relies on a per-protocol analysis, which maximizes the probability of finding a statistically significant difference between the two groups, i.e. of rejecting the hypothesis that has motivated the trial. Moreover, the ITT approach includes all individuals who were initially randomized, regardless of their adherence, and thus may dilute, or even mask, the true difference between the interventions in the case of non-adherence or losses to follow-up [59]. In the extreme, if most participants do not adhere to the assigned intervention or fail to complete the follow-up assessment, the ITT analysis will automatically show no difference. To prevent such paradoxes, our main analysis will rely on the perprotocol approach, i.e. include only participants who will have completed the one-year assessment. On the other hand, because of its popularity, we will use the ITT approach in "sensitivity analyses," as recommended by the CONSORT guidelines for non-inferiority trials [68]. Furthermore, our analyses will focus on (primary or secondary) outcomes at one year. Because all primary and secondary outcomes are measured on continuous scales, the methods outlined below for the analyses of the primary outcome will also apply to all secondary outcomes. However, the analysis of secondary outcomes will be exploratory in nature and some (e.g. for PFM function) may have more limited statistical power.

Preliminary descriptive analyses will compare the individuals in the two trial arms using means, medians, standard deviations, and interquartile ranges (IQR) for continuous variables and frequency distributions for categorical and binary variables. Any variable for which the difference between the two arms is considered clinically relevant will be adjusted for in the multivariable regression analyses (see below). The distribution of the outcome scores will be assessed for normality of residuals, using the Shapiro-Wilk test. In the case of significant violation of normality, appropriate parametric (e.g. logarithmic or Box-Cox) [77] or non-parametric [78] transformation will be applied, to meet the normality assumption underlying the univariate t-tests, as well as multivariable linear regression (see below).

Analyses for the primary outcome at one year (and secondary outcomes): The primary analysis will test our main hypothesis that group-based classes are not inferior to individualized treatment with respect to the primary outcome of relative (\%) reduction in the number of leakages at one year post randomization. (For all outcomes, the analyses outlined below will be repeated using data on post-intervention assessment.) As recommended for non-inferiority trials, the hypothesis of non-inferiority will be accepted if, and only if, the upper bound of the two-tailed (1-2 $\alpha)$ 95\% CI (corresponding to a conservative Type I error of 0.025 for the one-tailed independent-group $t$-test) for the mean difference (in \% reduction) excludes the non-inferiority threshold [68], set at $10 \%$ difference. This basic analysis will be extended to multivariable analyses. Specifically, two multivariable linear models, of increasing complexity, will be used to adjust the estimated difference between the \% reduction at one year in the two groups for, respectively, (1) only the two stratification variables (center and type of UI), as well as the baseline number of UI episodes (to account for regression to the mean phenomenon); and (2) (if necessary) in addition to variables in model (1): any variable, for which a clinically important imbalance between the two arms is revealed by descriptive analyses 
(see above). In both models, the two-way interactions between the trial arm and (a) center and (b) UI type, will be tested using 1-df model-based F-tests to verify if the difference between the effects of the two interventions depend on either of those stratification variables. In the case of a statistically significant interaction $(p<0.05$ for the F-test), the intervention effects will be estimated and tested separately in the respective subgroups [79]. The results of multivariable linear regression analyses will be summarized in terms of adjusted mean difference between the outcomes in the two trial arms, together with the 95\% model-based CIs. In sensitivity analyses for primary and secondary outcomes at one year, ITT analyses will be conducted, using the same statistical methods.

\section{Economic analysis}

Mean, median, and interquartile (semi-IQ) will be used to describe the costs for the two groups. Costeffectiveness analysis will be performed on the main outcome: reduction in number of leakages (\%) one year after randomization and expressed as a ratio of incremental costs and percentage of leakages. In order to verify the robustness of the economic analysis, a sensitivity analysis will be conducted on the hypothesis that the costs for each participant should be between the 25th and 75 th percentiles of the cost distribution.

\section{Multivariable models to predict response to treatment}

In additional analyses, multivariable logistic regression will be employed to assess patient characteristics associated with an increased likelihood of response to treatment, defined as at least $50 \%$ reduction in the number of UI episodes at one year. The logistic model will include, as independent variables, (a) the binary indicator of the trial arm, (b) the pre-intervention number of UI episodes, and (c) all previously listed covariates: age, BMI, general health status, level of physical activity, medical history and medications, parity and obstetrical history as well as type and duration of UI and current pad use. Independent, statistically significant predictors of response will be identified by $p<0.05$ for the respective multivariable model-based two-tailed 1-df Wald test and their effects will be summarized by adjusted odds ratios with $95 \%$ CIs.

\section{Discussion}

Considering demographic projections, human resources, and health system constraints, there is a pressing need to scale up evidence on the long-term benefits of lowcost interventions in urinary incontinent aging women and thereby improve clinical, functional, and social outcomes of this highly prevalent condition in this subpopulation. This GROUP trial, the first adequately powered $\mathrm{RCT}$, seeks to study the non-inferiority of group PFMT in comparison to individual one-on-one PFMT in aging women with UI. If group-based PFMT classes prove to be equally effective, as well as more cost-effective, compared to individual one-on-one PFMT sessions, this trial could have a positive impact on the accessibility of continence care for aging women in Canada.

The results of this RCT will be relevant to clinicians and clinical decision-makers as well as to administrative stakeholders. It will have implications for the organization and the administration of continence care services. Ultimately, the results may influence the cost of treatment per individual, the accessibility of conservative management, and the use of more invasive UI interventions such as medication and surgery in older UI women.

\section{Dissemination of study finding}

The results of this study will be disseminated through national and international scientific and professional conferences, in addition to undergraduate and postgraduate courses in PFM rehabilitation for physiotherapists.

\section{Trial status}

This trial is actively recruiting participants (353/364). The trial is ongoing and has a planned duration of five years, with recruitment running from September 2012 to March 2017.

\section{Additional files}

Additional file 1: Informed consent form. (PDF $82 \mathrm{~kb}$ )

Additional file 2: SPIRIT checklist. (DOC $120 \mathrm{~kb}$ )

\section{Abbreviations}

DBICl: Dowell-Bryant Incontinence Cost Index; ICIQ modules: International Consultation on Incontinence questionnaire modules; ICIQ-FLUTSex: ICIQFemale Lower Urinary Tract Symptoms sex; ICIQ-LUTSquol: ICIQ-Lower Urinary Tract Symptoms quality of life; ICIQ-N: ICIQ-Nocturia; ICIQ-UI short form: ICIQ-Urinary Incontinence short form; ICIQ-VS: ICIQ-Vaginal Symptoms; PFM: Pelvic floor muscle; PFMT: Pelvic floor muscle training;

$\mathrm{RCT}$ : Randomized controlled trial; UI: Urinary incontinence; US: Ultrasound

\section{Acknowledgements}

The authors wish to acknowledge the collaboration of the physiotherapists, research professionals contributing to recruitment, evaluations, and treatments of participants in this research project.

\section{Funding}

This study is supported by an operating grant from the Canadian Institute of Health Research (\#MSH-258993).

This study was also supported by two infrastructure grants (equipment) from the Canadian Foundation on Innovation (in Montreal: Dumoulin\#16209; in Sherbrooke; Morin\#29876).

Three of the four authors were supported by the following salary awards: Dr. Dumoulin: Tier II Canadian Research Chair in Urogynecological Health and Aging; Dr Mélanie Morin: research scholar award from the Fonds de Recherche du Québec en Santé; and Dr Marie-Hélène Mayrand: clinical research award from the Fonds de Recherche du Québec en Santé. The funders have no role in the study design, data collection, management, analysis and interpretation of data, writing the report or the decision to submit the report for publication. 


\section{Availability of data and materials}

Not applicable.

\section{Declarations}

This protocol has been prepare in accordance with Standard Protocol Items: Recommendations for Interventional Trials (SPIRIT), a completed SPIRIT checklist is included as Additional file 2.

\section{Authors' contributions}

$C D$ is the principal investigator and has directed the planning of the study design and developed assessment forms, study interventions, and database. She is in charge of data management and will take part in the analyzing and interpretation of data in addition to the submission of the reports for publication. She has the authority over the project. CD and MM coordinated the two study sites. MM, MHM, MT, and MA contributed to study conception, implementation, will take part in analysis and interpretation of data, and will help draft the manuscript. All authors will read and approve the final manuscripts. The present manuscript was primarily written by $C D$ and the statistical plan by MA. The rest of the authors have done critical revision of the manuscript. All authors approved the final version. Authorship for this and future publications will depend on each author contribution.

\section{Ethics approval and consent to participate}

This study has been evaluated and approved by the respective ethics committees at each of the five recruitment sites: the Research Ethics Committee of Institut Universitaire de Gériatrie de Montréal (CER IUGM 12-13-002), the Centre Hospitalier de l'Université de Montréal (CE 12.347), the CLSC Lucille Teasdale \& the CSSS Jeanne Mance (CSSSJM-2014-07-04), and the Centre Hospitalier de I'université de Sherbrooke (\#12-170-M5).

The consent form is mailed out to the participant after the telephone eligibility evaluation. Informed consent for each participant is obtained during the onsite eligibility evaluation by the evaluator. The participant has time to consider and ask questions to the evaluator before giving consent. There was no safety monitoring committee for this trial. None were required because of the low risk level of the provided intervention. However, participant safety is through the steering committee (composed of the trial's investigators) or monitored independently through the local ethics committees.

\section{Consent for publication}

Not applicable.

\section{Competing interests}

The authors declare that they have no competing interests.

\section{Publisher's Note}

Springer Nature remains neutral with regard to jurisdictional claims in published maps and institutional affiliations.

\section{Author details}

'School of Rehabilitation, Faculty of Medicine, Université de Montréal, Research Centre of the Institut Universitaire de Gériatrie de Montréal, 4565 Queen Mary M-5816, Montreal, QC H3W 1W5, Canada. ${ }^{2}$ School of Rehabilitation, Faculty of Medicine and Health Sciences, Université de Sherbrooke and Research Center of the Centre hospitalier universitaire de Sherbrooke (CHUS), Sherbrooke, QC, Canada. ${ }^{3}$ Department of Obstetrics and Gynecology and Social and Preventive Medicine, Université de Montréal and Research Center of the Centre Hospitalier de l'Université de Montréal, Montréal, QC, Canada. ${ }^{4}$ School of Rehabilitation, Faculty of Medicine and Health Sciences, Université de Sherbrooke and Research Center on Aging, Sherbrooke, QC, Canada. ${ }^{5}$ Department of Epidemiology, Biostatistics and Occupational Health, McGill University, Research Institute of the McGill University Health Centre, Montréal, QC, Canada.

Received: 13 March 2017 Accepted: 17 October 2017 Published online: 16 November 2017

\section{References}

1. Milsom I, Altman D, Cartwright R, Lapitan M, Nelson R, Sillen U, et al. Epidemiology of urinary incontinence $(\mathrm{UI})$ and other lower urinary tract symptoms (LUTS), pelvic organ prolapse (POP) and anal incontinence (AI).
In: Abrams P, Cardozo L, Khoury S, Wein A, editors. Incontinence. 5th ed Arnhem: European Association of Urology; 2013. p. 15-108.

2. Hunskaar $S$, Vinsnes $A$. The quality of life in women with urinary incontinence as measured by the sickness impact profile. J Am Geriatr Soc. 1991;39(4):378-82.

3. Johnson 2nd TM, Kincade JE, Bernard SL, Busby-Whitehead J, Hertz-Picciotto I, DeFriese GH. The association of urinary incontinence with poor self-rated health. J Am Geriatr Soc. 1998;46(6):693-9.

4. Ko Y, Lin SJ, Salmon JW, Bron MS. The impact of urinary incontinence on quality of life of the elderly. Am J Manag Care. 2005:11(4 Suppl):S103-11.

5. Temml C, Haidinger G, Schmidbauer J, Schatzl G, Madersbacher S. Urinary incontinence in both sexes: prevalence rates and impact on quality of life and sexual life. Neurourol Urodyn. 2000;19(3):259-71.

6. Sen I, Onaran M, Aksakal N, Acar C, Tan MO, Acar A, et al. The impact of urinary incontinence on female sexual function. Adv Ther. 2006:23(6):999-1008.

7. Wagg A, Chen L, Krischner-Hermans R, Kuchel G, Johnson T, Ostaszkeiwicz J, et al. Incontinence in the frail elderly. In: Abrams P, Cardozo L, Khoury S, Wein A, editors. Incontinence. 5th ed. Arnhem: European Association of Urology; 2013. p. 1001-101.

8. Imamura M, Abrams P, Bain C, Buckley B, Cardozo L, Cody J, et al. Systematic review and economic modelling of the effectiveness and cost-effectiveness of non-surgical treatments for women with stress urinary incontinence. Health Technol Assess. 2010;14(40):1-188. iii-iv.

9. Dumoulin C, Hay-Smith EJ, Mac Habee-Seguin G. Pelvic floor muscle training versus no treatment, or inactive control treatments, for urinary incontinence in women. Cochrane Database Syst Rev. 2014;5:CD005654.

10. Moore K, Dumoulin C, Berghmans B, Burgio K, Hagen S, N'Dow J, et al. Adult conservative management in Incontinence. In: Abrams P, Cardozo L, Khoury S, Wein A, editors. Incontinence. 5th ed. Arnhem: European Association of Urology; 2013.

11. Belisle S, Blake J, Basson R, Desindes S, Graves G, Grigoriadis S, et al. Canadian Consensus Conference on menopause, 2006 update. J Obstet Gynaecol Can. 2006;28(2 Suppl 1):S7-S94.

12. Canadian Consensus Conference on Urinary Incontinence: clinical practice guidelines for adults. http://www.continence-fdn.ca. Accessed 10 June 2016.

13. Swanson JG, Skelly J, Hutchison B, Kaczorowski J. Urinary incontinence in Canada. National survey of family physicians' knowledge, attitudes, and practices. Can Fam Physician. 2002:48:86-92.

14. Bland DR, Dugan E, Cohen SJ, Preisser J, Davis CC, McGann PE, et al. The effects of implementation of the Agency for Health Care Policy and Research urinary incontinence guidelines in primary care practices. J Am Geriatr Soc. 2003;51(7):979-84

15. The Canadian Continence Foundation. The impact of incontinence in Canada: A briefing document for policy-makers. Peterborough, ON: Canadian Continence Foundation; 2014.

16. Kim H, Yoshida H, Suzuki T. The effects of multidimensional exercise treatment on community-dwelling elderly Japanese women with stress, urge, and mixed urinary incontinence: a randomized controlled trial. Int J Nurs Stud. 2011:48(10):1165-72.

17. Sherburn M, Bird M, Carey M, Bo K, Galea MP. Incontinence improves in older women after intensive pelvic floor muscle training: an assessorblinded randomized controlled trial. Neurourol Urodyn. 2011;30(3):317-24.

18. Elliott V, de Bruin ED, Dumoulin C. Virtual reality rehabilitation as a treatment approach for older women with mixed urinary incontinence: a feasibility study. Neurourol Urodyn. 2015;34(3):236-43.

19. Borghuis M. Group remedial therapy in general practice for women with incontinence symptoms. Physiotherapy. 1994;87(5):235-41.

20. Demain S, Smith JF, Hiller L, Dziedzic K. Comparison of group and individual physiotherapy for female urinary incontinence in primary care. Physiotherapy. 2001;87(5):235-42

21. Cook T. Group treatment of female urinary incontinence. Physiotherapy. 2001;87(5):226-34

22. Martin C, Dumoulin C. Pelvic floor exercise classes for urinary incontinence in older women: a feasibility study. In: World Congress Physical Therapy abstract book. UK: Elsevier; 2007.

23. Kaapa EH, Frantsi K, Sarna S, Malmivaara A. Multidisciplinary group rehabilitation versus individual physiotherapy for chronic nonspecific low back pain: a randomized trial. Spine (Phila Pa 1976). 2006:31(4):371-6.

24. Labrie J, Berghmans BL, Fischer K, Milani AL, van der Wijk I, Smalbraak DJ, et al. Surgery versus physiotherapy for stress urinary incontinence. N Engl J Med. 2013;369(12):1124-33. 
25. Miller-Kovach $\mathrm{K}$, Hermann M, Winick $M$. The psychological ramifications of weight management. J Womens Health Gend Based Med. 1999;8(4):477-82.

26. Alewijnse D, Mesters I, Metsemakers J, van den Borne B. Predictors of longterm adherence to pelvic floor muscle exercise therapy among women with urinary incontinence. Health Educ Res. 2003;18(5):511-24.

27. Hay-Smith J, Dean S, Burgio K, McClurg D, Frawley H, Dumoulin C. Pelvicfloor-muscle-training adherence "modifiers": A review of primary qualitative studies-2011 ICS State-of-the-Science Seminar research paper III of IV. Neurourol Urodyn. 2015;34(7):622-31.

28. Locher JL, Goode PS, Roth DL, Worrell RL, Burgio KL. Reliability assessment of the bladder diary for urinary incontinence in older women. J Gerontol A Biol Sci Med Sci. 2001;56(1):M32-5.

29. Karantanis E, Allen W, Stevermuer TL, Simons AM, O'Sullivan R, Moore KH. The repeatability of the 24-hour pad test. Int Urogynecol J Pelvic Floor Dysfunct. 2005;16(1):63-8. discussion 68

30. Avery K, Donovan J, Peters TJ, Shaw C, Gotoh M, Abrams P. ICIQ: a brief and robust measure for evaluating the symptoms and impact of urinary incontinence. Neurourol Urodyn. 2004;23(4):322-30.

31. Abraham L, Hareendran A, Mills IW, Martin ML, Abrams P, Drake MJ, et al. Development and validation of a quality-of-life measure for men with nocturia Urology. 2004;63(3):481-6.

32. Kelleher CJ, Cardozo LD, Khullar V, Salvatore S. A new questionnaire to assess the quality of life of urinary incontinent women. Br J Obstet Gynaecol. 1997;104(12):1374-9.

33. Price N, Jackson SR, Avery K, Brookes ST, Abrams P. Development and psychometric evaluation of the ICIQ Vaginal Symptoms Questionnaire: the ICIQ-VS. BJOG. 2006;113(6):700-12.

34. Jackson S, Donovan J, Brookes S, Eckford S, Swithinbank L, Abrams P. The Bristol Female Lower Urinary Tract Symptoms questionnaire: development and psychometric testing. Br J Urol. 1996;77(6):805-12.

35. Tannenbaum C, Brouillette J, Korner-Bitensky N, Dumoulin C, Corcos J, le Tu $M$, et al. Creation and testing of the Geriatric Self-Efficacy Index for Urinary Incontinence. J Am Geriatr Soc. 2008;56(3):542-7.

36. Broome BA. Development and testing of a scale to measure self-efficacy for pelvic muscle exercises in women with urinary incontinence. Urol Nurs. 1999:19(4):258-68.

37. Yalcin I, Bump RC. Validation of two global impression questionnaires for incontinence. Am J Obstet Gynecol. 2003;189(1):98-101.

38. Laycock J. Assessment and treatment of pelvic floor dysfunction. Bradford: University of Bradford; 1990.

39. Dumoulin C, Bourbonnais D, Lemieux MC. Development of a dynamometer for measuring the isometric force of the pelvic floor musculature. Neurourol Urodyn. 2003;22(7):648-53.

40. Braekken IH, Majida M, Ellstrom-Engh M, Dietz HP, Umek W, Bo K. Test-retest and intra-observer repeatability of two-, three- and four-dimensional perineal ultrasound of pelvic floor muscle anatomy and function. Int Urogynecol J Pelvic Floor Dysfunct. 2008;19(2):227-35.

41. Dowell CJ, Bryant CM, Moore KH, Simons AM. Calculating the direct costs of urinary incontinence: a new test instrument. BJU Int. 1999;83(6):596-606.

42. Brubaker L, Nygaard I, Bo K, Tincello D, Homma Y, Cook J, et al. Research methodology. In: Abrams P, Cardozo L, Khoury S, Wein A, editors. Incontinence. 5th ed. Arnhem: European Association of Urology; 2013. p. 1863-95.

43. Ouslander JG, Hepps K, Raz S, Su HL. Genitourinary dysfunction in a geriatric outpatient population. J Am Geriatr Soc. 1986;34(7):507-14.

44. Burgio KL, Goode PS, Locher JL, Umlauf MG, Roth DL, Richter HE, et al. Behavioral training with and without biofeedback in the treatment of urge incontinence in older women: a randomized controlled trial. JAMA. 2002;288(18):2293-9.

45. Tannenbaum C, Bachand G, Dubeau CE, Kuchel GA. Experience of an incontinence clinic for older women: no apparent age limit for potential physical and psychological benefits. J Womens Health Gend Based Med. 2001;10(8):751-6.

46. Bradley CS, Rahn DD, Nygaard IE, Barber MD, Nager CW, Kenton KS, et al. The questionnaire for urinary incontinence diagnosis (QUID): validity and responsiveness to change in women undergoing non-surgical therapies for treatment of stress predominant urinary incontinence. Neurourol Urodyn. 2010;29(5):727-34.

47. Satskin D, Kelleher C, Avery K. Initial assessment in urinary and fecal incontinence in adult male and female patients. In: Abrams P, Cardozo L, Khoury S, Wein A, editors. Incontinence. 4th ed. Arnhem: European Association of Urology; 2009. p. 331-412.
48. Folstein MF, Folstein SE, McHugh PR. "Mini-mental state". A practical method for grading the cognitive state of patients for the clinician. J Psychiatr Res. 1975;12(3):189-98.

49. DiNubile NA. Strength training. Clin Sports Med. 1991;10(1):33-62.

50. Gunnarsson M, Teleman P, Mattiasson A, Lidfeldt J, Nerbrand C, Samsioe G. Effects of pelvic floor exercises in middle aged women with a history of naive urinary incontinence: a population based study. Eur Urol. 2002;41(5):556-61.

51. Dumoulin C, Chiva-Razavi S, Corriveau H. Is there a relationship between balance or lower extremity strength and the impact of mixed urinary incontinence in aging community-dwelling women?. In: International Continence Society Annual Congress: September 2009. San Francisco; 2009. https://www.ics.org/2009/abstract/112. Accessed 10 June 2016.

52. Morin M, Corriveau H, Dupuis K, Lefebvre I, Nadeau S, Filiatrault J, Dumoulin C. Identifying deficits in lower limb muscle strength, balance and mobility in elderly women suffering from urge and mixed urinary incontinence. Barcelona: ICS; 2013. https://www.ics.org/2013/abstract/562. Accessed 10 June 2016.

53. Dumoulin C, Lemieux MC, Bourbonnais D, Gravel D, Bravo G, Morin M. Physiotherapy for persistent postnatal stress urinary incontinence: a randomized controlled trial. Obstet Gynecol. 2004;104(3):504-10.

54. Bo K, Talseth T, Holme I. Single blind, randomised controlled trial of pelvic floor exercises, electrical stimulation, vaginal cones, and no treatment in management of genuine stress incontinence in women. BMJ. 1999;318(7182):487-93.

55. Madill SJ, Pontbriand-Drolet S, Tang A, Dumoulin C. Effects of PFM rehabilitation on PFM function and morphology in older women. Neurourol Urodyn. 2013;32(8):1086-95.

56. Addla S. Assessment of reliability of 1-day, 3-days and 7-days frequency volume charts. Eur Urol Suppl. 2004;2(30):165.

57. Abrams P, Cardozo L, Khoury S, Wein A. Incontinence. 5th ed. Arnhem: European Association of Urology; 2013.

58. Hilton P, Robinson D. Defining cure. Neurourol Urodyn. 2011;30(5):741-5.

59. Chen SY. The development and testing of the pelvic floor muscle exercise self-efficacy scale. J Nurs Res. 2004;12(4):257-66.

60. Srikrishna S, Robinson D, Cardozo L. Validation of the Patient Global Impression of Improvement (PGII) for urogenital prolapse. Int Urogynecol J. 2010;21(5):523-8.

61. Laycock J, Jerwood D. Pelvic Floor Muscle Assessment: The PERFECT Scheme. Physiotherapy. 2001;87(12):631-42.

62. Morin M, Dumoulin C, Gravel D, Bourbonnais D, Lemieux MC. Reliability of speed of contraction and endurance dynamometric measurements of the pelvic floor musculature in stress incontinent parous women. Neurourol Urodyn. 2007;26(3):397-403. discussion 404.

63. Dietz HP. Ultrasound imaging of the pelvic floor. Part I: two-dimensional aspects. Ultrasound Obstet Gynecol. 2004;23(1):80-92.

64. Dietz HP. Ultrasound imaging of the pelvic floor. Part II: three-dimensional or volume imaging. Ultrasound Obstet Gynecol. 2004;23(6):615-25.

65. Drummond M, O'Brian B, Stoddart G, Torrance G. Methods for the economic evaluation of health care programmes. 2nd ed. Oxford: Oxford University Press; 1997.

66. Simons AM, Dowell CJ, Bryant CM, Prashar S, Moore KH. Use of the Dowell Bryant Incontinence Cost Index as a post-treatment outcome measure after non-surgical therapy. Neurourol Urodyn. 2001;20(1):85-93.

67. Tousignant M, Moffet $H$, Nadeau S, Merette C, Boissy P, Corriveau H, et al. Cost analysis of in-home telerehabilitation for post-knee arthroplasty. J Med Internet Res. 2015;17(3):e83.

68. Piaggio G, Elbourne DR, Altman DG, Pocock SJ, Evans SJ, CONSORT Group. Reporting of noninferiority and equivalence randomized trials: an extension of the CONSORT statement. JAMA. 2006;295(10):1152-60.

69. Yalcin I, Peng G, Viktrup L, Bump RC. Reductions in stress urinary incontinence episodes: what is clinically important for women? Neurourol Urodyn. 2010;29(3):344-7.

70. Group CR, Martin DF, Maguire MG, Ying GS, Grunwald JE, Fine SL, et al. Ranibizumab and bevacizumab for neovascular age-related macular degeneration. N Engl J Med. 2011;364(20):1897-908.

71. Le Henanff A, Giraudeau B, Baron G, Ravaud P. Quality of reporting of noninferiority and equivalence randomized trials. JAMA. 2006;295(10):1147-51.

72. de Oliveira CF, Rodrigues AM, Arruda RM, Ferreira Sartori MG, Girão MJBC, Castro RA. Pelvic floor muscle training in female stress urinary incontinence: comparison between group training and individual treatment using PERFECT assessment scheme. Int Urogynecol J. 2009;20(12):1455-62. 
73. Lamb SE, Pepper J, Lall R, Jorstad-Stein EC, Clark MD, Hill L, et al. Group treatments for sensitive health care problems: a randomised controlled trial of group versus individual physiotherapy sessions for female urinary incontinence. BMC Womens Health. 2009:9:26.

74. Janssen CC, Lagro-Janssen AL, Felling AJ. The effects of physiotherapy for female urinary incontinence: individual compared with group treatment BJU Int. 2001;87(3):201-6.

75. Goode PS, Burgio KL, Locher JL, Roth DL, Umlauf MG, Richter HE, et al. Effect of behavioral training with or without pelvic floor electrical stimulation on stress incontinence in women: a randomized controlled trial. JAMA. 2003:290(3):345-52.

76. Hintze J. PASS. Kaysville: NCSS, LLC; 2008. https://www.ncss.com/. Accessed 10 June 2016.

77. Box G, Cox D. An analysis of transformations. J R Stat Soc Ser B. 1964;26(2):211-52.

78. Ramsay J. Monotone regression splines in action (with discussion). Stat Sci. 1988;:3:425-61

79. Abrahamowicz M, Beauchamp ME, Fournier P, Dumont A. Evidence of subgroupspecific treatment effect in the absence of an overall effect: is there really a contradiction? Pharmacoepidemiol Drug Saf. 2013;22(11):1178-88.

Submit your next manuscript to BioMed Central and we will help you at every step:

- We accept pre-submission inquiries

- Our selector tool helps you to find the most relevant journal

- We provide round the clock customer support

- Convenient online submission

- Thorough peer review

- Inclusion in PubMed and all major indexing services

- Maximum visibility for your research

Submit your manuscript at www.biomedcentral.com/submit
Biomed Central 